\title{
Envisager le futur énergétique : des aspirations sociétales aux enjeux techniques
}

Nadia Maïzi, mathématicienne, Mines ParisTech, et François Briens, docteur en mathématiques appliquées

\author{
Arbitrant entre plusieurs stratégies, voire choix de société, les modèles permettent l'exploration \\ de différents scénarios pour aider à prendre des décisions. Une famille de modèles permet \\ de comparer en détail trois scénarios, respectivement basés sur une croissance dite "verte » \\ de la consommation d'énergie, avec augmentation ou suppression progressive du nucléaire, \\ et sur une décroissance de la consommation d'énergie.
}

En ce qui concerne les enjeux énergétiques de long terme, la question de la pertinence des orientations technologiques se pose. Les modèles issus de la famille TIMES (The Integrated MARKAL-EFOM System) [1] permettent de réaliser des exercices prospectifs dans ce cadre. Proposant une description détaillée des technologies disponibles, ces modèles, pilotés par un scénario d'évolution de la demande à l'horizon 2050, évaluent l'offre technologique de façon à minimiser le cout total actualisé du système énergétique sur l'horizon considéré.

Pour alimenter de tels modèles, il faut aborder la question suivante : comment évoluera la demande énergétique au cours des 50 prochaines années? Question complexe, car les usages de l'énergie sont variés : se chauffer, se transporter, se distraire, produire... En outre, ils sont conditionnés à la fois par les infrastructures, les comportements, les choix de consommation, et plus largement par les modes de vie et le type d'organisation sociale ${ }^{(a)}$. Aussi, pour explorer les répercussions de différents choix de société (intégrant différentes options concernant le nucléaire) et de modes de vie sur la demande énergétique, nous avons développé un modèle de simulation macroéconomique [2] pour le périmètre français ${ }^{(b)}$.

Voyons comment la déclinaison de nos modèles permet l'éclairage de deux options préconisées comme alternatives aux tendances actuelles (voir encadré, p. 51). La première s'inscrit dans la perspective d'une croissance verte. Elle se présente comme un pari technologique :innovation et progrès technique y jouent un rôle critique, à la fois moteurs et fruits de la croissance économique, et également sources espérées de solutions à l'épuisement des ressources naturelles. Traduite dans notre modèle macroéconomique [2], cette stratégie conduit à une diminution de la consommation d'électricité de 15\% entre 2012 et 2050.

La deuxième option explorée vise à refléter la perspective d'acteurs des mouvements de la décroissance. Elle constitue plutôt un pari anthropologique : celui d'une révolution culturelle se traduisant par une évolution profonde des valeurs, normes, comportements, des modes de vie, de l'organisation sociale, et une mutation des systèmes de besoins vers une plus grande sobriété. Pour décrypter cette ambition, une série d'entretiens a été réalisée auprès d'acteurs proches des mouvements de la décroissance, chaque entretien étant ensuite traduit en scénario. Nous proposons ici d'analyser le scénario reflétant la plus volontariste des visions recueillies lors des entretiens. Notre modélisation macroéconomique [2] indique que ce scénario implique une diminution de la demande électrique de $56 \%$ entre 2012 et 2050 .
Ces deux scénarios de demande électrique, assortis de contraintes spécifiques, sont utilisés pour élaborer l'offre technologique du secteur électrique, optimale économiquement, jusqu'à l'horizon 2050.

Le scénario de croissance verte sera envisagé selon deux options : une première option ouvre la possibilité d'investir dans de nouvelles capacités nucléaires ; une seconde poursuit une politique de sortie du nucléaire et l'objectif d'atteindre un mix électrique $100 \%$ renouvelable en 2050. Dans ce dernier cas, le prolongement de 20 ans de la durée de vie des réacteurs existants, moyennant surcout, est autorisée. Le scénario de décroissance préconise une hypothèse de sortie du nucléaire sans possibilité de prolongement au-delà de la durée de vie des réacteurs (40 ans). Pour prolonger l'esprit de sobriété technologique sous-jacent, ni les technologies d'effacement, permettant de différer la demande de consommation électrique (comme les réseaux optimisés, dits smartgrids), ni les nouvelles technologies de stockage $^{(c)}$ dédiées à la production d'électricité ne sont autorisées.

Le premier élément d'arbitrage mis en évidence par les résultats de notre modèle (fig. 1) est le niveau des exportations d'électricité qui décroit drastiquement dans les deux options "100\% renouvelable". Si la part du nucléaire évolue selon les hypothèses retenues, les niveaux d'investissement en capacité de production 


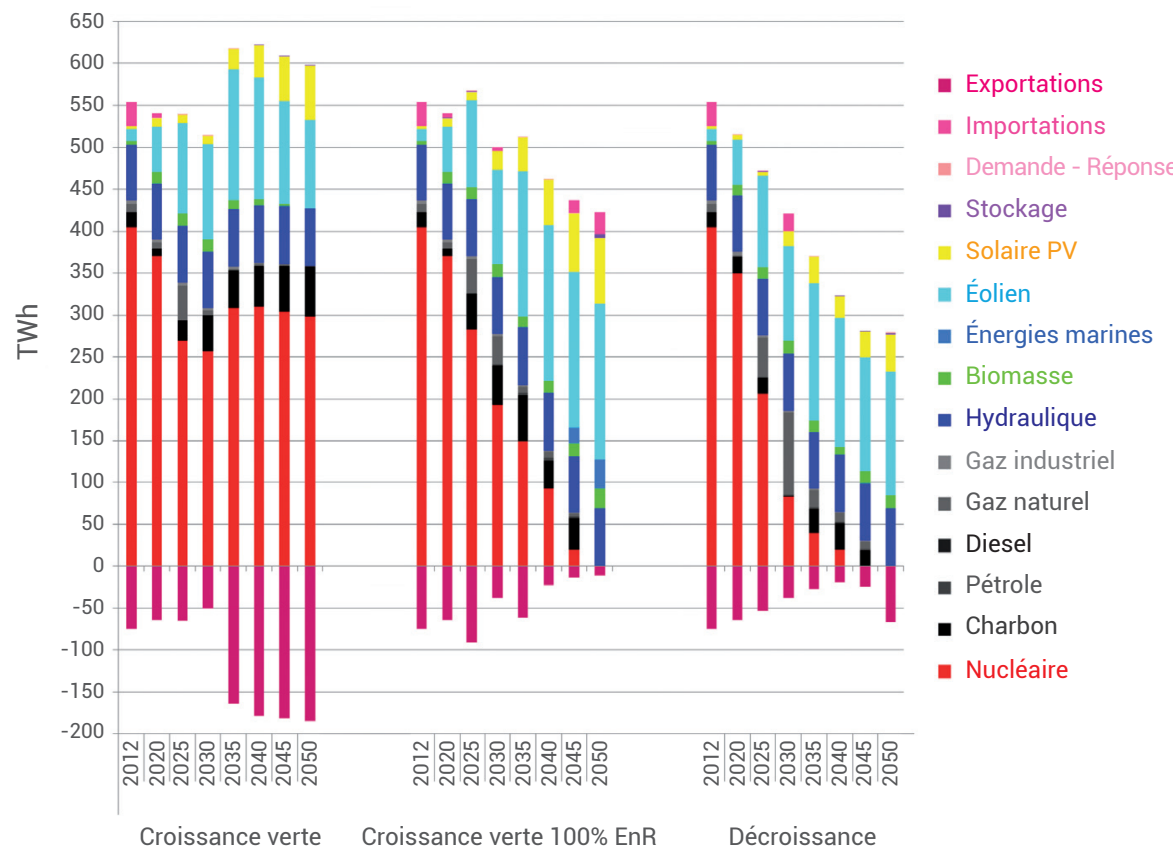

1. Différentes parts de la production électrique, en fonction du temps, dans les scénarios de croissance " verte » avec ou sans nucléaire, et de décroissance.

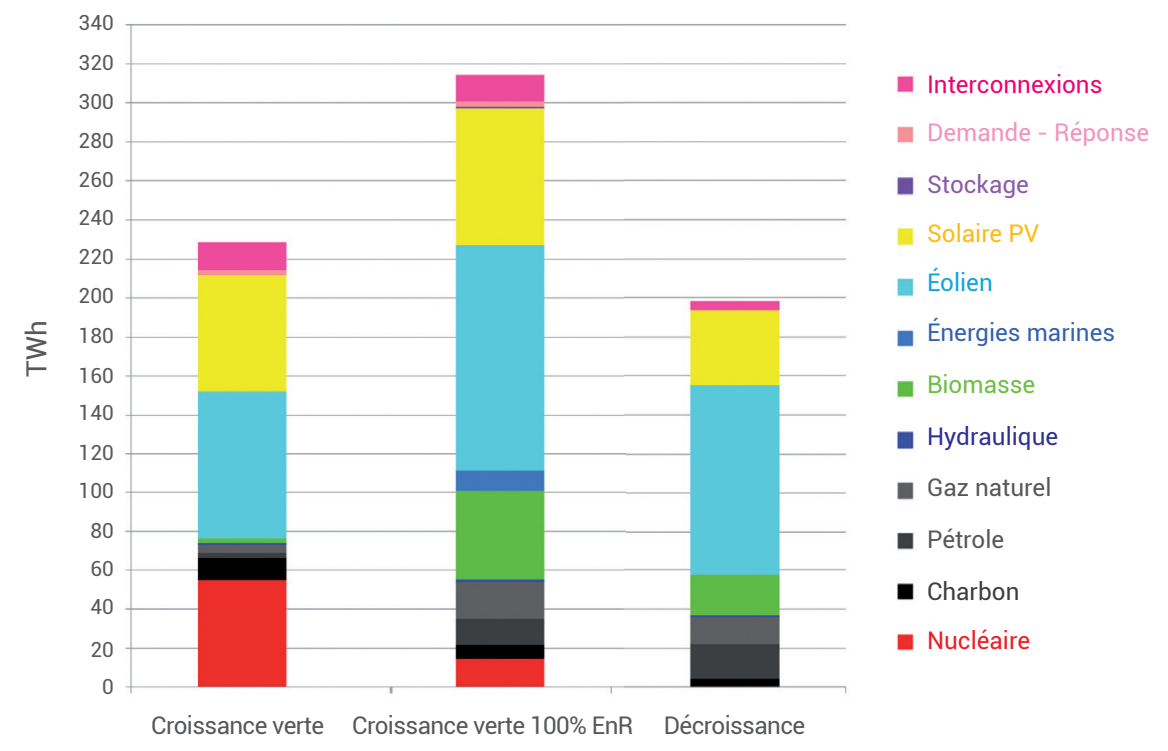

2. Capacités de production électrique installées d'ici 2050 selon les trois scénarios.

\section{L'indicateur cinétique}

Sous réserve que le synchronisme soit assuré à l'échelle du réseau $[5,6]$, cet indicateur correspond au temps d'épuisement de l'énergie cinétique ${ }^{(d)}$ embarquée dans le système électrique rapportée à la fluctuation maximale envisageable soit en consommation (écart au pic), soit en perte de génération. II s'exprime comme

$$
\frac{E_{\text {cin }}}{\max \left(\Sigma_{k} S_{k}, P_{\text {pic }}-\Sigma_{k} S_{k}\right)}
$$

où $E_{c i n}$ est l'énergie cinétique répartie sur le réseau, $\Sigma_{k} S_{k}$ est la puissance apparente maximum fournie par les générateurs avant fluctuation, et $P_{\text {pic }}$ le pic de la puissance appelée.

\section{\〉}

électrique sont contrastés : le scénario "croissance verte $100 \%$ renouvelable " arrive en tête pour le cumul de nouvelles capacités installées sur l'horizon d'étude (fig. 2). Les deux scénarios de sortie du nucléaire font appel à des technologies reposant sur l'usage de ressources fossiles. Ce résultat, récurrent dans nos études [3], indique qu'au-delà de la question du démantèlement, une sortie du nucléaire devra être accompagnée de façon volontariste pour limiter le recours à des technologies fortement émettrices. Les différences sensibles de niveaux d'investissement entre les scénarios se répercutent sur le cout total actualisé du système électrique : le passage aux scénarios plus sobres en émissions de gaz à effet de serre entraine, par rapport au scénario croissance verte, un surcout de $16 \%$ pour le cas d'une croissance verte $100 \%$ renouvelable et une diminution de cout de $4,5 \%$ dans le scénario de décroissance.

Tandis qu'un mix de production électrique donné semble satisfaire à des critères environnementaux, la question reste entière sur sa capacité à satisfaire aux opérations système, afin d'éviter une rupture de la fourniture d'électricité par déséquilibre entre approvisionnement et demande (black-out). Pour aborder ce problème qui met en jeu des phénomènes transitoires de l'ordre de la seconde voire de la milliseconde, nous avons élaboré un indicateur [4] qui permet d'estimer le temps nécessaire pour que le système recouvre un état de fonctionnement satisfaisant suite à une perturbation d'ampleur significative (voir ci-contre). Pour garantir la fiabilité du système électrique, un niveau minimum ${ }^{(\mathrm{e})}$ de cet indicateur doit être maintenu correspondant au temps de recours à la réserve primaire dont le rôle est de retrouver un équilibre entre production et consommation - en dehors de considérations de régime nominal ou d'optimum économique. Or on constate (fig. 3) que la valeur de l'indicateur se dégrade (par rapport à sa référence 2012) pour les deux scénarios à objectif «100\% renouvelables ». En effet, avec la pénétration du renouvelable variable, les technologies appelées ne disposent pas (photo-voltaïque) ou peu (éolien) d'inertie mécanique. La fiabilité du système électrique se trouve donc, dans ces deux scénarios, fortement dégradée.

Loin d'invalider les options analysées et leur vocation à limiter les émissions du système électrique, ces résultats incitent à 


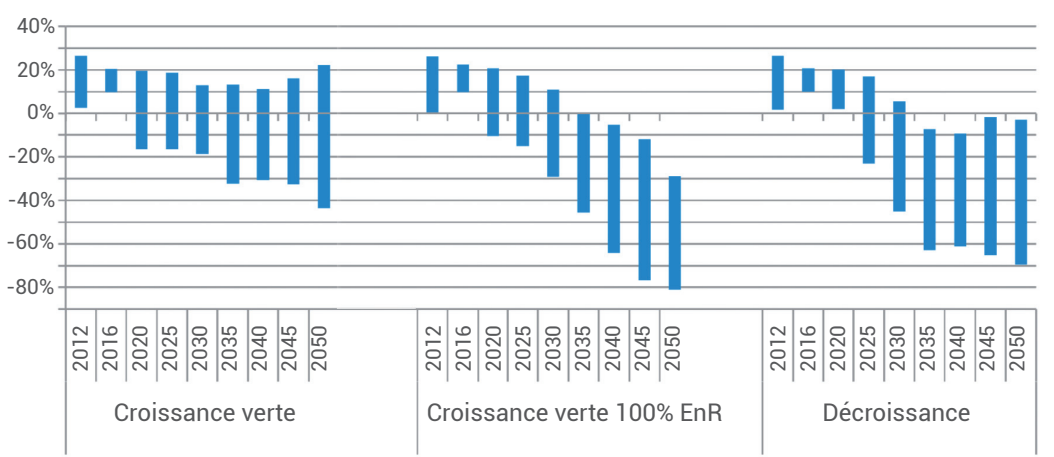

3. Indicateur cinétique en fonction du temps, dans les trois scénarios. Plus cet indicateur est haut, plus le réseau est stable par rapport aux fluctuations. La valeur indiquée est la valeur relative par rapport au minimum constaté en 2012.

réfléchir à la mise en adéquation des solutions prônées avec les exigences d'opération du système. À travers une étude du cas de l'ile de la Réunion à l'horizon 2030, nous avons pu montrer qu'un design technologique répondant à la contrainte de maintien du niveau de l'indicateur cinétique, permet d'envisager un mix $100 \%$ renouvelable $[6,7]$ autorisant à la fois une pénétration de plus de $50 \%$ des ressources intermittentes et une diminution des nouvelles capacités installées.

Les outils de prospective que nous avons développés, illustrés à travers ces analyses, mettent en relief les conséquences possibles mais parfois éludées, de certains choix, qu'ils soient techniques, liés à des évolutions comportementales, à des modes de vie ou d'organisation sociale. En permettant ainsi un débat informé, ils offrent au politique " désireux d'une aventure calculée » [8], les moyens de sa réalisation. a. Dont les choix démographiques.

b. Pour une étude de scénarios à l'échelle mondiale, voir dans ce dossier l'article de S. Bouneau (p. 46).

c. Par exemple les batteries et super-condensateurs haute capacité, les stockages thermiques.

d. Énergie cinétique due à la rotation des parties mécaniques.

e. De l'ordre de quelques dizaines de secondes, ce temps est de $40 \mathrm{~s}$ en moyenne pour la France en 2013, et de 25 s pour une ile comme La Réunion en 2008 : plus l'indicateur est élevé, plus le système peut faire face à des fluctuations importantes.

f. Le taux annuel d'amélioration de l'efficacité énergétique est supposé deux fois supérieur au taux moyen observé en France pour chaque branche sur la période 1996-2012.

g. Les gains marginaux d'efficacité énergétique sont supposés de plus en plus faibles et nuls après 2050 .

\section{Références}

1. R. Loulou, G. Goldstein, K. Noble, "Documentation of the MARKAL family of models", Energy Technology Systems Analysis Program (2004).

2. F. Briens, «La Décroissance au prisme de la modélisation prospective : Exploration macroéconomique d'une alternative paradigmatique ", Thèse de Mines ParisTech (2015)

3. N. Maïzi, E. Assoumou, "Future prospects for nuclear power in France", Applied Energy 136 (2014), 849-859, http://dx.doi.org/10.1016/j.apenergy.2014.03.056

4. M. Drouineau, N. Maïzi, V. Mazauric, "Impacts of intermittent sources on the quality of power supply: The key role of reliability indicators", Applied Energy 116 (2014) 333-343, https://doi.org/10.1016/j. apenergy.2013.11.069

5. V. Krakowski, X. Li, V. Mazauric, N. Maïzi, "Power system synchronism in planning exercise: From Kuramoto lattice model to kinetic energy aggregation", Energy Procedia 105 (2017) 2712-2717, https://doi.org/ 10.1016/j.egypro.2017.03.921

6. N. Maïzi, V. Mazauric, E. Assoumou, S. Bouckaert, V. Krakowski, X. Li, P. Wang, "Maximizing intermittency in $100 \%$ renewable and reliable power systems: A holistic approach applied to Reunion Island in 2030", Applied Energy 227 (2018) 332-341, http://doi. org/10.1016/j.apenergy.2017.08.058

7. S. Bouckaert, P. Wang, V. Mazauric, N. Maïzi, "Expanding renewable energy by implementing dynamic support through storage technologies", Energy Procedia 61 (2014), 2000-2003, http://dx.doi.org/10.1016/j.egypro. 2014.12.061

8. P. Massé, Le plan ou l'anti-hasard, Gallimard (1965).

\section{Croissance « verte » et décroissance}

\section{Croissance verte}

La taille des ménages continue de décroitre tendanciellement, la consommation finale augmente, la mobilité à longue distance continue de se développer, la mobilité locale se reporte partiellement sur les transports en commun, les véhicules électriques se diffusent rapidement, l'économie poursuit de façon modérée sa tertiarisation, le secteur résidentiel bénéficie d'un rythme de rénovation thermique élevé, et les hypothèses de progrès technique et d'amélioration de l'efficacité énergétique des processus de production et des équipements sont très fortes ${ }^{(f)}$.

\section{Décroissance}

La consommation des ménages change de composition et décroit significativement du fait de l'adoption progressive de modes de vie "frugaux " et du développement des pratiques de mise en commun, la mobilité longue distance diminue fortement, les déplacements se reportent en grande partie sur des modes plus doux (vélo, transports en commun, train, etc.), l'économie est relocalisée, l'agriculture devient essentiellement "biologique ", les rénovations thermiques dans le secteur résidentiel sont limitées, et les hypothèses d'amélioration de l'efficacité énergétique des processus de production et des équipements des ménages sont très modestes $(\mathrm{g})$. 